\title{
A New Numerical Approach of MHD Flow with Heat and Mass Transfer for the UCM Fluid over a Stretching Surface in the Presence of Thermal Radiation
}

\author{
S. Shateyi ${ }^{1}$ and G. T. Marewo ${ }^{2}$ \\ ${ }^{1}$ Department of Mathematics, University of Venda, Private Bag X5050, Thohoyandou 0950, South Africa \\ ${ }^{2}$ Department of Mathematics, University of Swaziland, Private Bag 4, Kwaluseni, Swaziland
}

Correspondence should be addressed to S. Shateyi; stanford.shateyi@univen.ac.za

Received 18 March 2013; Accepted 13 August 2013

Academic Editor: Tirivanhu Chinyoka

Copyright (c) 2013 S. Shateyi and G. T. Marewo. This is an open access article distributed under the Creative Commons Attribution License, which permits unrestricted use, distribution, and reproduction in any medium, provided the original work is properly cited.

This paper numerically investigates the magnetohydrodynamic boundary layer flow with heat and mass transfer of an incompressible upper-convected Maxwell fluid over a stretching sheet in the presence of viscous dissipation and thermal radiation as well as chemical reaction. The governing partial differential equations are transformed into a system of ordinary differential equations by using suitable similarity transformations. The resultant highly nonlinear ordinary differential equations are then solved using spectral relaxation method. The results are obtained for velocity, temperature, concentration, skin friction, and Nusselt number. The effects of various material parameters on the flow with heat and mass transfer and the dimensionless variables are illustrated graphically and briefly discussed.

\section{Introduction}

In the past few decades, the studies of boundary layer flows of Newtonian and non-Newtonian fluids of stretching surfaces have received great attention by virtue of their numerous applications in the fields of metallurgy, chemical engineering, and biological systems. These applications include geothermal reservoirs, wire and fiber coating, food stuff processing, reactor fluidization, transpiration cooling, enhanced oil recovery, packed bed catalytic reactors, and cooling of nuclear reactors. The prime aim in extrusion is to keep the surface quality of the extricate. Coating processes demand a smooth glossy surface to meet the requirements for the best appearance and optimum properties. Sakiadis $[1,2]$ did pioneering work on boundary layer flow on a continuously moving surface. After that many investigators discussed various aspects of the stretching flow problem (see, e.g., Chiam [3], Crane [4], Liao and Pop [5], Khan and Sanjayanand [6], Abel and Mahesha [7], and Fang et al. [8], among others).
A number of industrial fluids such as molten plastics, artificial fibers, blood, polymetric liquids, and food stuff exhibit non-Newtonian fluid behaviour. In many industrial processes, cooling continuous strips or filaments is done by drawing them through a quiescent fluid. It must be noted that during these processes, the strips are sometimes stretched or shrunk. Therefore the properties of the final product depend to a great extent on the rate of cooling. The rate of cooling can be controlled and the desired characteristics of the final product can be obtained by drawing the strips in electrically conducting fluids subjected to uniform magnetic fields.

In recent years, MHD flows of viscoelastic fluids above stretching sheets have also been studied by various researchers (Liu [9], Cortell [10], among others). This is the simplest subclass of viscoelastic fluid known as the second grade fluid. However, a non-Newtonian second grade fluid does not give meaningful industrial results for highly elastic fluids such as polymer melts, which occur at high Deborah number (Hayat et al. [11]). For theoretical results to become 
of any industrial use, more realistic viscoelastic fluid models such as upper-convected Maxwell model should be used in the analysis.

Alizadeh-Pahlavan et al. [12] investigated using a twoauxiliary-parameter homotopy analysis method for the problem of laminar MHD flow of an upper-convected Maxwell fluid above a porous isothermal stretching sheet. Aliakbar et al. [13] analyzed the influence of Maxwell fluids above stretching sheets. Abel et al. [14] performed an analysis to investigate the influence of MHD and thermal radiation on the two-dimensional steady flow of an incompressible, upper-convected Maxwell fluid. Motsa et al. [15] investigated the MHD boundary layer flow of an incompressible upperconvected Maxwell fluid over a porous stretching surface. Hayat et al. [16] investigated MHD flow of a more realistic viscoelastic fluid model above a porous stretching sheet. Sadeghy et al. [17] also studied MHD flows of upperconvected Maxwell model fluids above porous stretching sheet using homotopy analysis method. Raftari and Yildrim [18] approximated an analytical solution of the magnetohydrodynamic boundary layer flow of an upper-convected Maxwell fluid over a porous stretching sheet. More recently, Alinejad and Samarbakhsh [19] numerically investigated the flow and heat transfer characteristics of the incompressible viscous flow over a nonlinear stretching sheet with viscous dissipation.

Hayat et al. [20] proposed the modified decomposition method and the Padé approximants for the MHD flow over a nonlinear sheet. In recent years, scientists and engineers have endeavored to develop more accurate and fast converging numerical and/or analytical techniques. Methods such as homotopy and their hybrid techniques have been extensively applied to solve nonlinear equations (Liao [21], He [22], Domairry and Nadim [23], Shateyi and Motsa [24], Sheikholeslami et al. [25], and Ganji [26], among others). Recently, successive linearization method has been reported and successfully utilized in solving boundary value problems (see, e.g., Motsa and Shateyi [27], Motsa et al. [28], and Shateyi and Motsa [29]).

The aim of this study is to investigate the effects of thermal radiation and viscous dissipation on steady MHD flow with heat and mass transfer of an upper-convected Maxwell fluid past a stretching sheet in the presence of a chemical reaction. Secondly, we aim to use a recently developed iterative method known as spectral relaxation method (SRM), and details of this method can be found in [30-32].

\section{Mathematical Formulation}

We consider the steady and incompressible MHD boundary layer flow with heat and mass transfer of an electrically conducting fluid obeying UCM model over a stretching sheet in the presence of thermal radiation. The flow is generating by the stretching of the sheet by applying two equal and opposite forces along the $x$-axis, keeping the origin fixed and considering the flow to be confined to the region $y>0$. We assume that the continuous stretching sheet has a linear velocity, $u=b x$, with $b$ as the stretching rate and $x$ being the distance from the slit. We impose a uniform magnetic field of strength $B_{0}$ along the $y$-axis, and the induced magnetic field is negligible. This assumption is valid on a laboratory scale under the assumption of small magnetic Reynolds number, and the external electric field is zero. We also assume that the boundary layer approximations are applicable to all momentum, energy, and mass equations. Although this theory is incomplete for viscoelastic fluids, but it is more plausible for Maxwell fluids as compared to other viscoelastic fluid models (Renardy [33]). Following Sadeghy et al. [17] among others, in a two-dimensional flow, the equation of continuity, the equation of motion, and the diffusion equations can be written as

$$
\begin{gathered}
\frac{\partial u}{\partial x}+\frac{\partial v}{\partial y}=0 \\
u \frac{\partial u}{\partial x}+v \frac{\partial u}{\partial y}+\lambda_{1}\left[u^{2} \frac{\partial^{2} u}{\partial x^{2}}+v^{2} \frac{\partial^{2} u}{\partial y^{2}}+2 u v \frac{\partial^{2} u}{\partial x \partial y}\right] \\
=v \frac{\partial^{2} u}{\partial y^{2}}-\frac{\sigma B_{0}^{2}}{\rho} u \\
u \frac{\partial T}{\partial x}+v \frac{\partial T}{\partial y}=\frac{k}{\rho c_{p}} \frac{\partial^{2} T}{\partial y^{2}}+\frac{\mu}{\rho c_{p}}\left(\frac{\partial u}{\partial y}\right)^{2}-\frac{1}{\rho c_{p}} \frac{\partial q_{r}}{\partial y} \\
u \frac{\partial C}{\partial x}+v \frac{\partial C}{\partial y}=D \frac{\partial^{2} C}{\partial y^{2}}-K_{n}\left(C-C_{\infty}\right)^{n}
\end{gathered}
$$

where $u$ and $v$ are velocity components in the $x$ - and $y$-directions, respectively, $\rho$ is the fluid density, $\sigma$ is the electrical conductivity, $B_{0}$ is the uniform magnetic field, $T$ is the temperature, $c_{p}$ is the specific heat at constant pressure, $k$ is the thermal conductivity, $C$ is the concentration of the species diffusion, $D$ is the diffusion coefficient of the diffusion species, $v$ is the kinematic viscosity, $\lambda$ is the relaxation time, and $K_{n}$ denotes the reaction rate constant of the $n$ th-order homogeneous and irreversible reaction. The appropriate boundary conditions are

$$
\begin{gathered}
u(x, 0)=U_{w}(x)=b x, \\
v(x, 0)=0, \\
T(x, 0)=T_{\infty}+T_{s}\left(\frac{x}{l}\right)^{2}, \\
C(x, 0)=C_{\infty}+C_{s}\left(\frac{x}{l}\right)^{2}, \\
u(x, \infty)=0, \\
T(x, \infty)=T_{\infty}, \\
C(x, \infty)=C_{\infty} .
\end{gathered}
$$

By using the Rosseland diffusion approximation (Hossain et al. 1999, among other researchers), the radiative heat flux, $q_{r}$, is given by

$$
q_{r}=-\frac{4 \sigma^{*}}{3 K_{s}} \frac{\partial T^{4}}{\partial y}
$$


where $\sigma^{*}$ and $K_{s}$ are the Stefan-Boltzmann constant and the Rosseland mean absorption coefficient, respectively. We assume that the temperature differences within the flow are sufficiently small such that

$$
T^{4} \approx 4 T_{\infty}^{3} T-3 T_{\infty}^{4}
$$

Using (6) and (7) in the last term of (3) we obtain

$$
\frac{\partial q_{r}}{\partial y}=-\frac{16 \sigma^{*} T_{\infty}^{3}}{3 K_{s}} \frac{\partial^{2} T}{\partial y^{2}}
$$

2.1. Similarity Transformation. To make the problem amenable we introduce the following nondimensional quantities:

$$
\begin{gathered}
\eta=\sqrt{\frac{b}{v} y,} \quad \psi=\sqrt{b \nu} \times f(\eta) \\
\theta(\eta)=\frac{T-T_{\infty}}{T_{w}-T_{\infty}} \\
\phi(\eta)=\frac{C-C_{\infty}}{C_{w}-C_{\infty}}
\end{gathered}
$$

where $f(\eta)$ is the dimensionless stream function and $\eta$ is the similarity variable, $\theta$ is the dimensionless temperature, and $\phi$ is the mass concentration. The continuity equation is automatically satisfied through the variables. Then introducing the relations (9) into (2)-(3), we obtain the following nonlinear system of ordinary differential equations:

$$
\begin{gathered}
f^{\prime \prime \prime}+f f^{\prime \prime}-f^{\prime 2}-M^{2} f^{\prime}+\beta\left(2 f f^{\prime} f^{\prime \prime}-f^{2} f^{\prime \prime \prime}\right)=0, \\
\left(1+\frac{4}{3} R\right) \theta^{\prime \prime}+\operatorname{Pr}\left(f \theta^{\prime}-2 f^{\prime} \theta\right)+\operatorname{Pr} \operatorname{Ec} f^{\prime \prime 2}=0, \\
\phi^{\prime \prime}+\operatorname{Sc}\left[f \phi^{\prime}-2 f^{\prime} \phi-\gamma \phi^{n}\right]=0 .
\end{gathered}
$$

Here $M^{2}=\sigma B_{0}^{2} / \rho b$ and $\beta=\lambda b$ are magnetic and elastic parameters, respectively, $\operatorname{Pr}=\mu c_{p} / k$ is the Prandtl number, $\mathrm{Ec}=b l^{2} / c_{p} T_{s}$ is the Eckert number, $R=4 \sigma^{*} T_{\infty}^{3} / k K_{s}$ is the thermal radiation parameter, $\gamma=K_{n} C_{0}^{n-1} b^{n-2} x^{n} / l^{2}$ is the chemical reaction parameter, and $\mathrm{Sc}=D / \nu$ is the Schmidt number. The boundary conditions are

$$
\begin{aligned}
& f(0)=0, \quad f^{\prime}(0)=1, \quad \theta(0)=1, \quad \phi(0)=1, \\
& f^{\prime} \longrightarrow 0, \quad \theta \longrightarrow 0, \quad \phi \longrightarrow 0, \quad \text { as } \eta \longrightarrow \infty .
\end{aligned}
$$

\section{Method of Solution}

The Successive Relaxation Method (SRM) begins by letting

$$
p=f^{\prime}
$$

so that $p^{\prime}=f^{\prime \prime}$ and $p^{\prime \prime}=f^{\prime \prime \prime}$. Consequently, (10) through (12) become

$$
\begin{gathered}
p^{\prime \prime}+f p^{\prime}-p^{2}-M^{2} p+\beta\left(2 f p p^{\prime}-f^{2} p^{\prime \prime}\right)=0, \\
\left(1+\frac{4}{3} R\right) \theta^{\prime \prime}+\operatorname{Pr}\left(f \theta^{\prime}-2 p \theta\right)+\operatorname{Pr} \operatorname{Ec} p^{\prime 2}=0, \\
\phi^{\prime \prime}+\operatorname{Sc}\left[f \phi^{\prime}-2 p \phi-\gamma \phi^{n}\right]=0 .
\end{gathered}
$$

Proceeding in a manner similar to the Gauss-Seidel method, (14) and (15) through (16) are replaced by the following recursive formulae:

$$
\begin{gathered}
f_{r+1}^{\prime}=p_{r}, \quad f_{r+1}(0)=0, \\
p_{r+1}^{\prime \prime}+f_{r+1} p_{r+1}^{\prime}-p_{r}^{2}-M^{2} p_{r+1} \\
+\beta\left(2 f_{r+1} p_{r} p_{r}^{\prime}-f_{r+1}^{2} p_{r}^{\prime \prime}\right)=0, \\
p_{r+1}(0)=1, \quad p_{r+1}(\infty)=0, \\
\frac{1}{\operatorname{Pr}}\left(1+\frac{4 R}{3}\right) \theta_{r+1}^{\prime \prime}+f_{r+1} \theta_{r+1}^{\prime} \\
-2 p_{r+1} \theta_{r+1}+\operatorname{Ec} p_{r+1}^{\prime 2}=0, \\
\theta_{r+1}(0)=1, \quad \theta_{r+1}(\infty)=0, \\
\phi^{\prime \prime}+\operatorname{Sc}\left[f \phi_{r+1}^{\prime}-2 p_{r+1} \phi_{r+1}-\gamma \phi_{r}^{n}\right]=0, \\
\phi_{r+1}(0)=1, \quad \phi_{r+1}(\infty)=0 .
\end{gathered}
$$

The Chebyshev spectral collocation method will be used to solve (18) through (21). First, we replace the semi-infinite interval $[0, \infty)$ with the closed interval $[0, L]$, where $L$ is sufficiently large. It is convenient to use the change of variable

$$
\eta(\xi)=\frac{1+\xi}{2} L
$$

to map the interval $[0, L]$ on the $\eta$-axis onto the interval $[-1,1]$ on the $\xi$-axis. On $[-1,1]$ we form a computational grid $-1=\xi_{N}<\xi_{N-1}<\cdots<\xi_{0}=1$, where

$$
\xi_{j}=\cos \left(\frac{\pi p}{N}\right), \quad j=0,1, \ldots, N,
$$

are the Chebyshev collocation points. The derivative $h^{\prime}\left(\xi_{j}\right)$ at each collocation point is evaluated using formula

$$
\begin{aligned}
& h^{\prime}\left(\xi_{j}\right)=\sum_{k=0}^{N} D_{j k} h\left(\xi_{k}\right) \\
& \Longrightarrow \mathbf{h}^{\prime}=D \mathbf{h},
\end{aligned}
$$

where $D$ is the Chebyshev differentiation matrix. Successive application of Chebyshev differentiation reveals the more general formula

$$
\frac{d^{q}}{d \xi^{q}} \mathbf{h}=D^{q} \mathbf{h}
$$


Chebyshev differentiation transforms (18) through (21) to discrete form:

$$
\begin{array}{ccc}
A_{1} \mathbf{f}_{r+1}=B_{1}, \quad f_{r+1}\left(\xi_{N}\right)=0, & p_{r+1}\left(\xi_{0}\right)=0, \\
A_{2} \mathbf{p}_{r+1}=B_{2}, & p_{r+1}\left(\xi_{N}\right)=1, & \theta_{r+1}\left(\xi_{0}\right)=0, \\
A_{3} \boldsymbol{\Theta}_{r+1}=B_{3}, & \theta_{r+1}\left(\xi_{N}\right)=1, & \phi_{r+1}\left(\xi_{0}\right)=0, \\
A_{4} \boldsymbol{\Phi}_{r+1}=B_{4}, & \phi_{r+1}\left(\xi_{N}\right)=1,
\end{array}
$$

where

$$
\begin{gathered}
A_{1}=D, \quad B_{1}=\mathbf{p}_{r}, \\
A_{2}=D^{2}+\operatorname{diag}\left[\mathbf{f}_{r+1}\right] \mathrm{D}-M^{2} I, \\
B_{2}=\mathbf{p}_{r}^{2}-\beta\left(2 \mathbf{f}_{r+1} \mathbf{p}_{r} \mathbf{p}_{r}^{\prime}-\mathbf{f}_{r+1}^{2} \mathbf{p}_{r}^{\prime \prime}\right), \\
A_{3}=\left(1+\frac{4}{3} R\right) D^{2}+\operatorname{Pr}\left(\operatorname{diag}\left[\mathbf{f}_{r+1}\right] D-2 \operatorname{diag}\left[\mathbf{p}_{r+1}\right]\right), \\
B_{3}=-\operatorname{PrEc} \mathbf{p}_{r+1}^{\prime 2}, \\
A_{4}=D^{2}+\operatorname{Sc}\left(\operatorname{diag}\left[\mathbf{f}_{r+1}\right] D-2 \operatorname{diag}\left[\mathbf{p}_{r+1}\right]\right), \\
B_{4}=\operatorname{Sc} \gamma \mathbf{\Phi}_{r}^{n} .
\end{gathered}
$$

Initial approximations needed to drive this iterative scheme must satisfy boundary conditions (13). Suitable choices are

$$
\begin{array}{cc}
f_{0}(\eta)=1-e^{-\eta}, & p_{0}(\eta)=e^{-\eta}, \\
\theta_{0}(\eta)=e^{-\eta}, & \phi_{0}(\eta)=e^{-\eta} .
\end{array}
$$

\section{Results and Discussion}

In this section we give the SRM results for the main parameters that have significant effects on the fluid flow velocity and temperature. We remark that all the SRM results presented in this work were obtained using $N=50$ collocation points, and also convergence was achieved after as few as five iterations. Also the infinity value $\left(\eta_{\infty}\right)$ was taken to be 50 . It is also important to note that the magnetic field is taken quite strong by assigning large values of $M$ to ensure the occurrence of steady flow near the sheet. Unless otherwise stated, the default values for the parameters are taken as $M=1, \beta=0.1, R=1$, $\operatorname{Pr}=0.71, \mathrm{Ec}=0.1, \mathrm{Sc}=0.2, \gamma=0.2$, and $n=2$. In order to validate the numerical method, it was compared with the MATLAB routine $b v p 4 c$ which is an adaptive Lobatto quadrature iterative scheme. Table 1 presents a comparison between SRM approximate results and the $b v p 4 c$ results for selected default values of the magnetic parameter $M$. It can be seen from this table that there is an excellent agreement between the results from the two methods. Analyzing Table 1 shows that an increase in the magnetic field strength leads to an increase in the skin-friction but a decrease to the Nusselt number. This is physically expected as application of a transverse magnetic field produces a drag force which then reduces the flow velocity but generates heat within the fluid.
TABLE 1: Comparison of the SRM results of $-f^{\prime \prime}(0),-\theta^{\prime}(0)$ with those obtained by $b v p 4 c$ for different values of the magnetic parameter.

\begin{tabular}{lcccc}
\hline & \multicolumn{2}{c}{$-f^{\prime \prime}(0)$} & \multicolumn{2}{c}{$-\theta^{\prime}(0)$} \\
$M$ & $b v p 4 c$ & SRM & $b v p 4 c$ & SRM \\
\hline 0.0 & 1.42811186 & 1.42811186 & 0.57932373 & 0.57932373 \\
1.0 & 1.87763199 & 1.87763199 & 0.40978550 & 0.40978550 \\
2.0 & 2.71897650 & 2.71897650 & 0.27809236 & 0.27809236 \\
\hline
\end{tabular}

TABLE 2: Comparison of the SRM results of $-f^{\prime \prime}(0),-\theta^{\prime}(0)$ with those obtained by $b v p 4 c$ for different values of the elastic parameter.

\begin{tabular}{lcccc}
\hline & \multicolumn{2}{c}{$-f^{\prime \prime}(0)$} & \multicolumn{2}{c}{$-\theta^{\prime}(0)$} \\
$\beta$ & $b v p 4 c$ & SRM & $b v p 4 c$ & SRM \\
\hline 0.0 & 1.39382112 & 1.39382112 & 3.22830067 & 3.22830067 \\
0.2 & 1.46105462 & 1.46105462 & 3.20679682 & 3.20679682 \\
0.4 & 1.52344386 & 1.52344386 & 3.18653107 & 3.18653107 \\
\hline
\end{tabular}

Table 2 gives a comparison of the SRM results to those obtained by the $b v p 4 c$ for different values of the elasticity parameter $\beta$. We again observe that the results from the two methods agree very well giving confidence to the current proposed method. Increasing the fluid elasticity parameter leads to the increase in the skin friction coefficient but a decrease in the heat transfer coefficient. Figures 1 and 2 show the influence of the elasticity parameter $\beta$ on the $u$-velocity and $v$-velocity profiles, respectively. From both these figures, we observe that an increase in the elasticity parameter results in the decrease in both velocity components and a decrease in the thickness of the momentum boundary layer.

Figures 3 and 4 depict the effects of the magnetic field parameter $M$ on the $u$-velocity and $v$-velocity profiles, respectively. From these figures we clearly observe that increasing magnetism significantly reduces the thickness of the boundary layer, thereby reducing the velocity components. Physically, the application of the transverse magnetic field presents a damping effect on the flow velocity by producing a drag force that opposes the fluid motion.

Figure 5 depicts the effect of increasing the elasticity parameter $\beta$ on the temperature distribution. A decrease in the streamwise velocity component, $u$, can result in a decrease in the amount of heat transferred on the surface sheet. Similarly, a decrease in the transverse velocity component, $v$, means that the amount of fresh fluid which is extended from the lower-temperature region outside the boundary layer and directed towards the sheet is reduced, thereby reducing the rate of heat transfer. These two effects on the velocity components in the same direction reinforce each other. Thus, an increase in the elastic number increases the temperature distribution in the fluid as depicted in Figure 5.

Figure 6 represents the dimensionless temperature for different values of the magnetic field parameter $M$. From this figure we clearly see that the temperature profiles increase with the increase of the magnetic field parameter. Thus the applied magnetic field tends to heat the fluid and thus reduces 


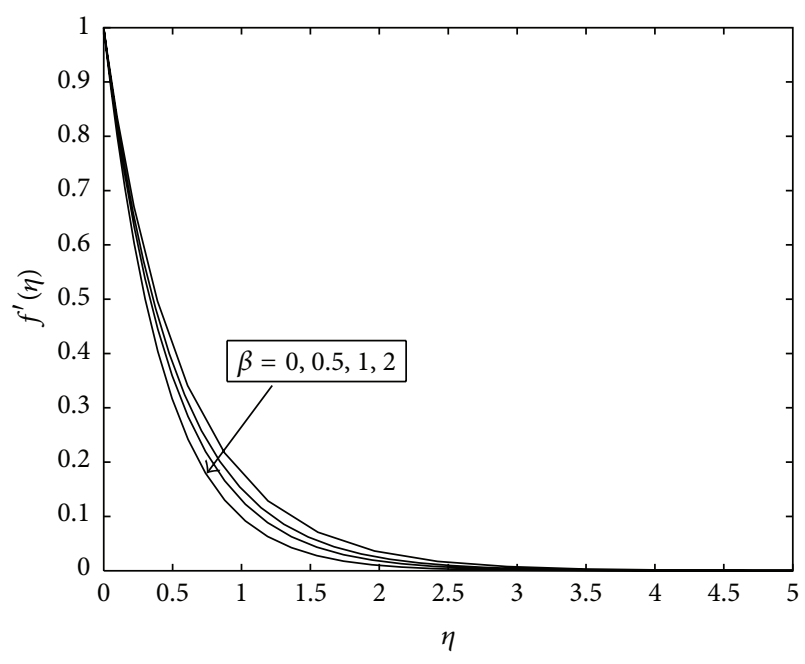

FIGURE 1: Graph of the SRM solutions of the $u$-velocity for different values of $\beta$.

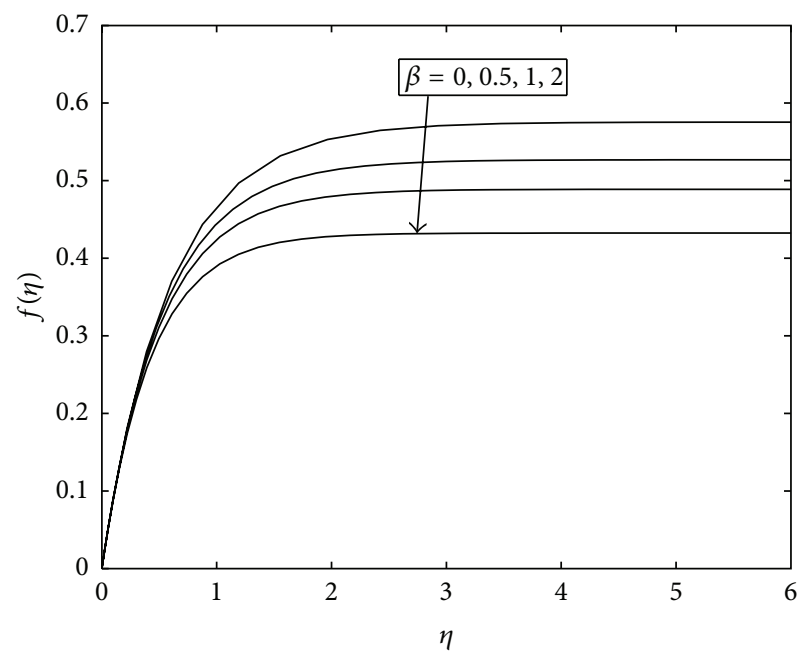

FIGURE 2: Effect of the $\beta$ on the $v$-velocity profile.

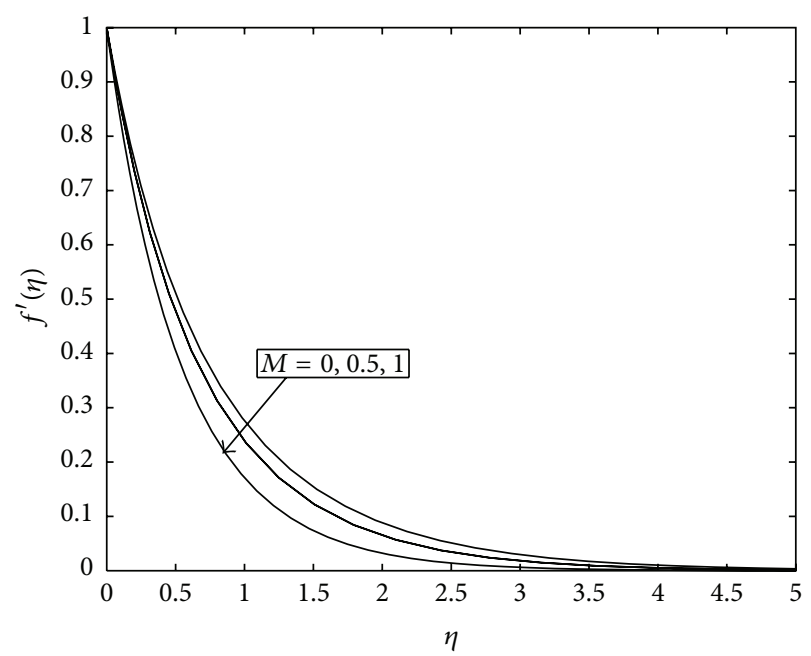

FIGURE 3: Influence of the magnetic parameter $M$ on the $u$-velocity profile.

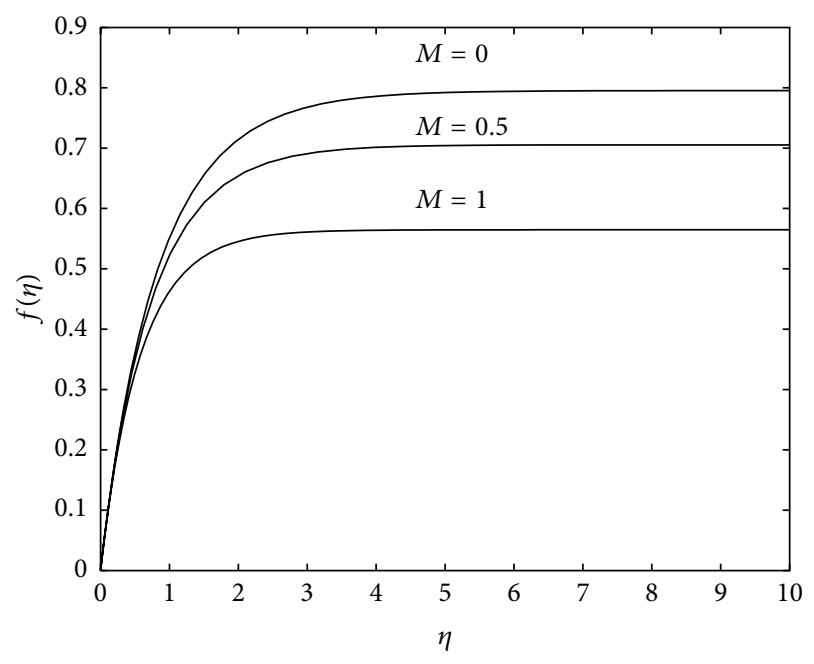

FIGURE 4: The variation of the $v$-velocity component for different values of $M$.

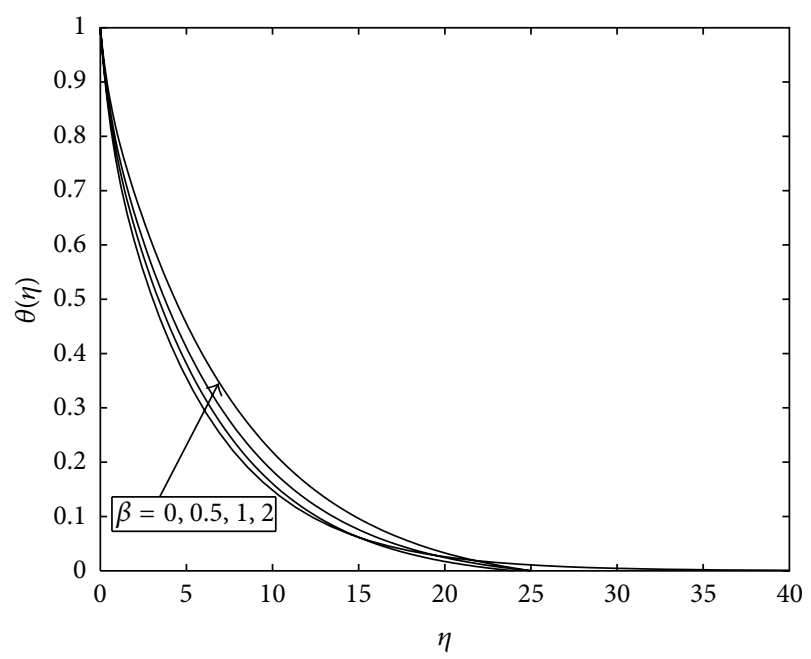

FIGURE 5: Graph of the SRM solutions of the temperature distribution for different values of $\beta$.

the heat transfer from the wall which in turn enlarges the thermal boundary layer thickness.

In Figure 7 we display the effect of the Eckert number on the temperature profiles. An increase in the values of the Eckert number is seen to increase the temperature of the fluid at any point above the sheet. Increasing the Eckert number allows energy to be stored in the fluid region as a consequence of dissipation due to viscosity and elastic deformation.

The influence of thermal radiation on the temperature profiles is shown in Figure 8. It can be seen that the thermal boundary layer thickness increases as $R$ increases. This induces the decrease in the absolute value of the temperature gradient at the surface. Thus, the heat transfer rate at the surface decreases with increasing $R$, thereby causing the temperature profiles to increase.

Figure 9 depicts the effect of increasing the Prandtl number on the fluid temperature distribution. An increase in 


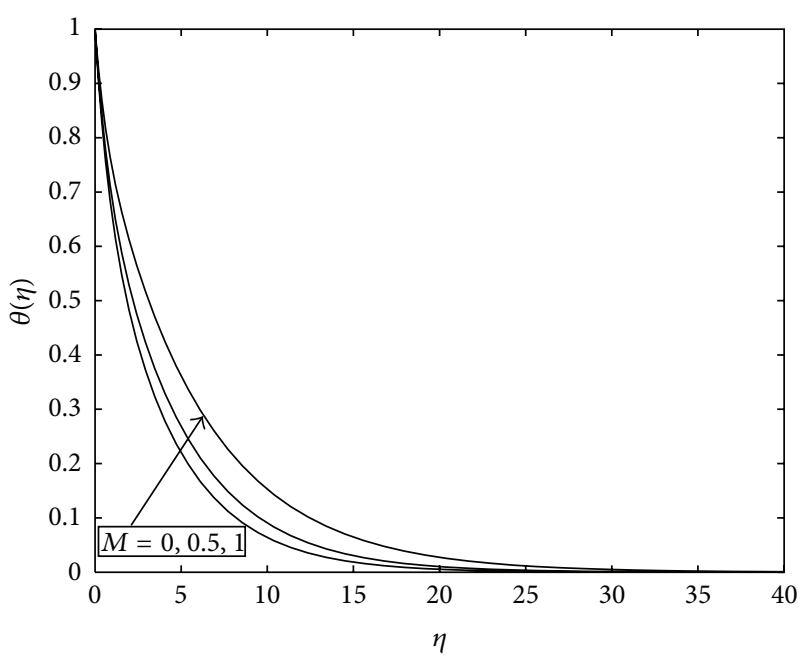

FIGURE 6: Variation of the temperature distribution for different values of $M$.

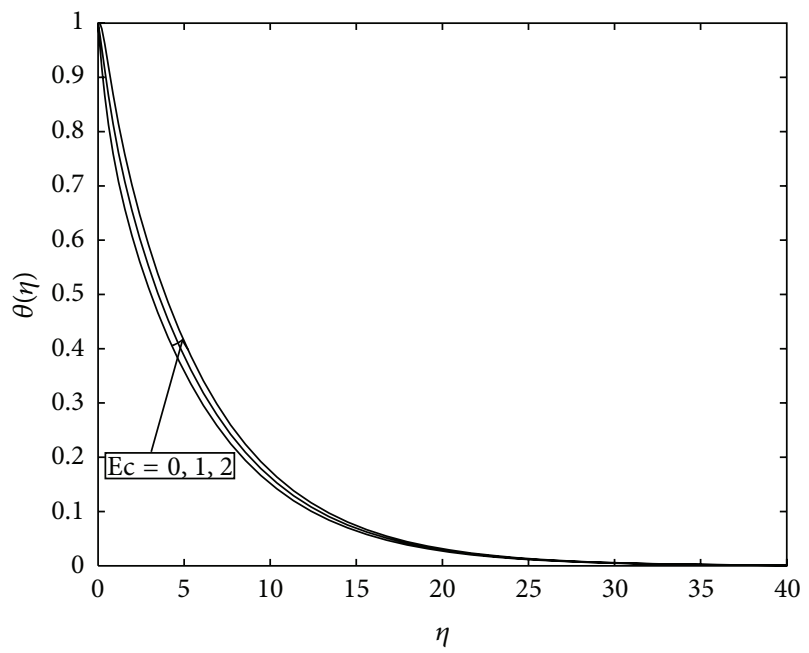

FIGURE 7: Graph of the SRM solutions of the temperature profile for different values of Ec.

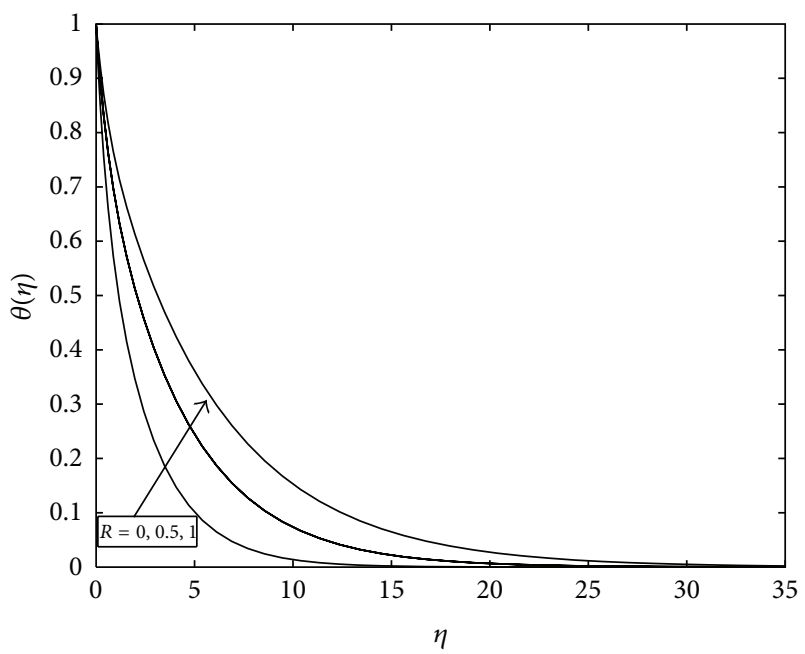

FIGURE 8: Influence of thermal radiation of the fluid temperature.

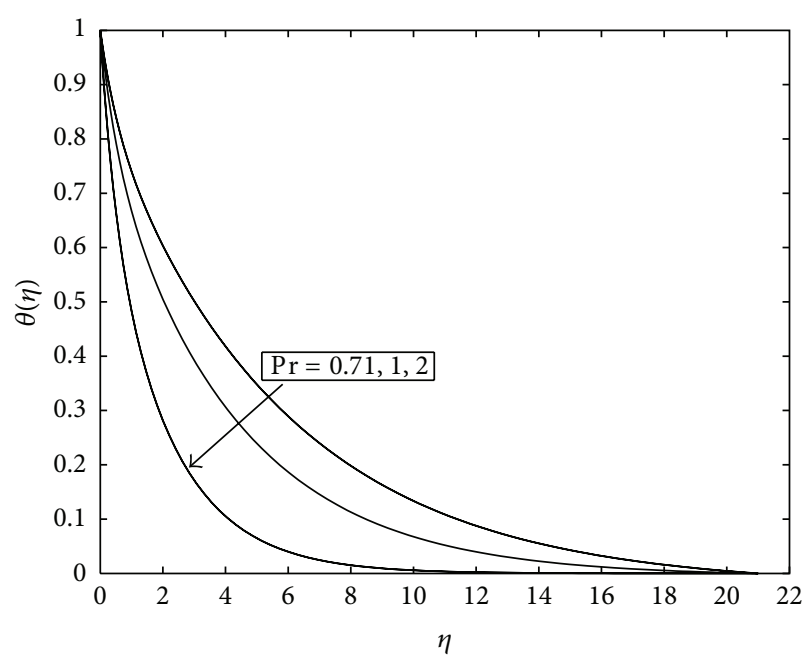

FIgure 9: The effect of the Prandtl number on the temperature distribution.

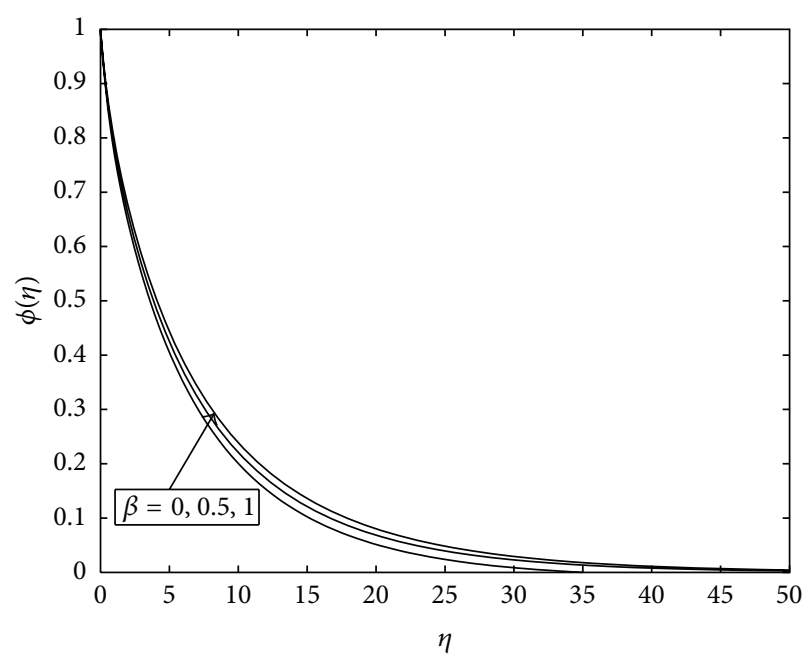

FIGURE 10: Graph of the SRM solutions of the concentration profile for different values of $\beta$.

the Prandtl number as expected is seen to reduce the fluid temperature above the sheet. This is because as the Prandtl number increases, the thermal boundary layer becomes thinner. Thus the rate of thermal diffusion drops, resulting in the fluid temperature dropping as well.

Figure 10 shows how the elastic parameter $\beta$ affects the concentration profiles. As the elasticity parameter reduces the flow velocity, it means that less fluid is taken away at any given point resulting in the concentration profiles increasing. The same phenomenon happens when the values of the magnetic parameter increase as can be clearly seen in Figure 11. The reduction of flow velocity as the result of increasing the strength of the magnetic field causes the fluid concentration to increase as less fluid is taken downstream at any given point.

Figure 12 presents the profiles of the concentration for selected default values of the chemical reaction parameter 


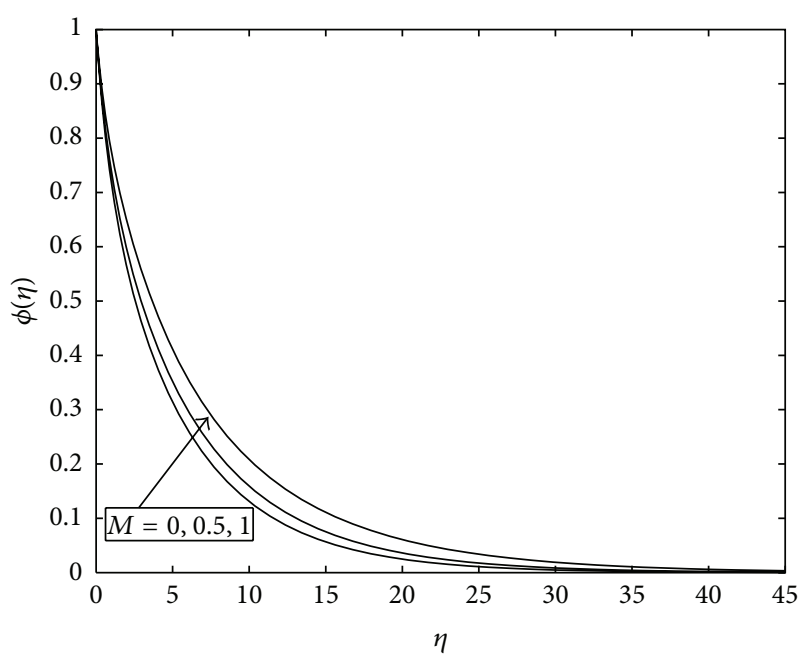

FIGURE 11: Graph of how the magnetic parameter affects the concentration distribution.

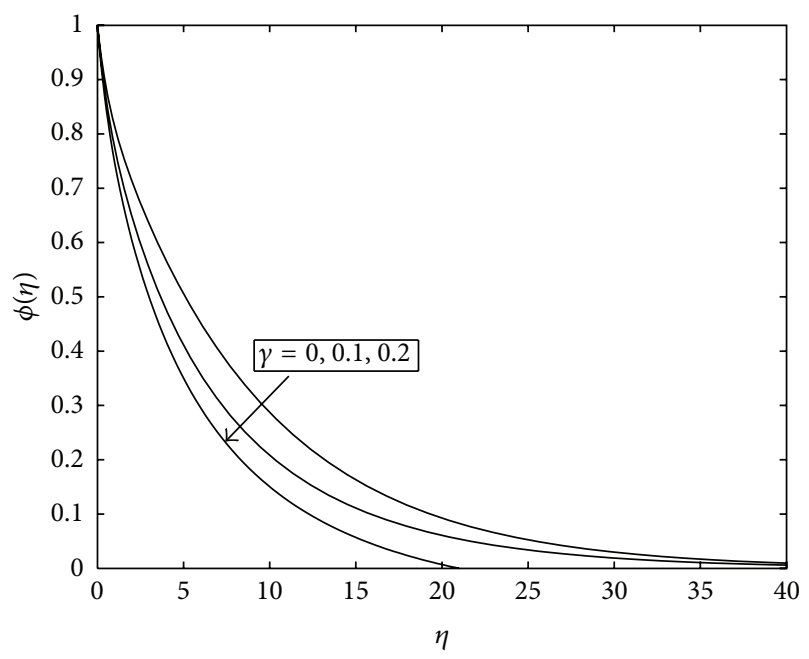

FIGURE 12: Influence of the chemical reaction on the concentration distribution.

$\gamma \geq 0$. It is observed from this figure that an increase in the values of chemical reaction parameter leads to a decrease in the concentration profiles. The concentration boundary layer becomes thin as the reaction parameter increases.

Figure 13 shows graphically the effect of increasing the reaction-order parameter $n$. The effect of $n$ is seen as to increase the fluid concentration.

\section{Conclusion}

The present work analyzed the MHD flow with heat and mass transfer within a boundary layer of an upper-convected Maxwell fluid above a stretching sheet in the presence of viscous dissipation, thermal radiation, and chemical reaction. Numerical results are presented in tabular/graphical form to elucidate the details of flow with heat and mass transfer characteristics and their dependence on the various physical

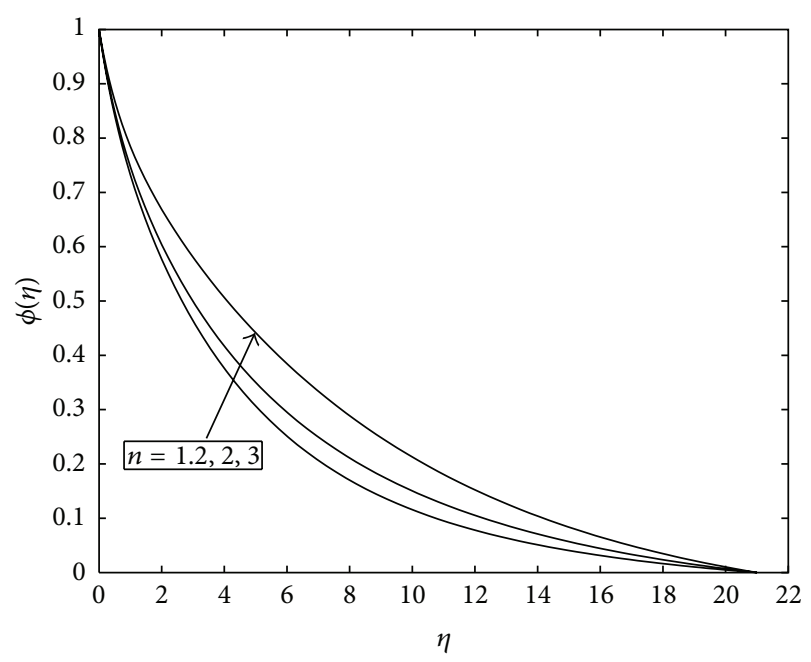

FIGURE 13: Graph of the SRM solutions of the concentration for different orders of the homogeneous reaction.

parameters. The accuracy of the SRM is validated against the MATLAB in-built $b v p 4 c$ routine for solving boundary value problems.

(1) We observe that the flow velocity is decreased when the magnetic parameter increases. Also an increase in the elasticity parameter results in velocity decrements. However, both the temperature and concentration profiles are enhanced by increasing the values of the magnetic parameter as well as the elasticity parameter.

(2) The dimensionless temperature $\theta$ increases with increase in the thermal radiation but decreases with increasing Prandtl number.

(3) The effect of the chemical reaction $\gamma$ is to decrease the fluid concentration while the concentration profiles are increased as the order of reaction $n$ is increased.

(4) It is shown that the skin-friction increases with an increase of the magnetic parameter and elasticity parameter, but the Nusselt number and Sherwood number (not shown for brief) are found to be decreased as these parameters are increased.

(5) Finally the Nusselt number increases with increasing values of the Prandtl number but decreases as thermal radiation increases.

\section{Acknowledgments}

The authors wish to acknowledge financial support from the University of Venda and NRF.

\section{References}

[1] B. C. Sakiadis, "Boundary-layer behaviour on continuous solid surfaces: I. Boundary-layer equations for two-dimensional and axisymmetric flow," AIChE Journal, vol. 7, no. 1, pp. 26-28, 1961. 
[2] B. C. Sakiadis, "Boundary-layer behaviour on continuous solid surfaces: II. Boundary-layer equations for two-dimensional and axisymmetric flow," AIChE Journal, vol. 7, no. 2, pp. 221-225, 1961.

[3] T. C. Chiam, "Hydromagnetic flow over a surface stretching with a power-law velocity," International Journal of Engineering Science, vol. 33, no. 3, pp. 429-435, 1995.

[4] L. J. Crane, "Flow past a stretching plate," Zeitschrift für Angewandte Mathematik und Physik, vol. 21, no. 4, pp. 645-647, 1970.

[5] S. Liao and I. Pop, "Explicit analytic solution for similarity boundary layer equations," International Journal of Heat and Mass Transfer, vol. 47, no. 1, pp. 75-85, 2004.

[6] S. K. Khan and E. Sanjayanand, "Viscoelastic boundary layer flow and heat transfer over an exponential stretching sheet," International Journal of Heat and Mass Transfer, vol. 48, no. 8, pp. 1534-1542, 2005.

[7] M. S. Abel and N. Mahesha, "Heat transfer in MHD viscoelastic fluid flow over a stretching sheet with variable thermal conductivity, non-uniform heat source and radiation," Applied Mathematical Modelling, vol. 32, no. 10, pp. 1965-1983, 2008.

[8] T. G. Fang, C. F. F. Lee, and J. Zhang, "The boundary layers of an unsteady incompressible stagnation-point flow with mass transfer," International Journal of Non-Linear Mechanics, vol. 46, no. 7, pp. 942-948, 2011.

[9] I. C. Liu, "Flow and heat transfer of an electrically conducting fluid of second grade in a porous medium over a stretching sheet subject to a transverse magnetic field," International Journal of Non-Linear Mechanics, vol. 40, no. 4, pp. 465-474, 2005.

[10] R. Cortell, "Flow and heat transfer of an electrically conducting fluid of second grade over a stretching sheet subject to suction and to a transverse magnetic field," International Journal of Heat and Mass Transfer, vol. 49, no. 11-12, pp. 1851-1856, 2006.

[11] T. Hayat, Z. Abbas, and M. Sajid, "Series solution for the upperconvected Maxwell fluid over a porous stretching plate," Physics Letters A, vol. 358, no. 5-6, pp. 396-403, 2006.

[12] A. Alizadeh-Pahlavan, V. Aliakbar, F. Vakili-Farahani, and K. Sadeghy, "MHD flows of UCM fluids above porous stretching sheets using two-auxiliary-parameter homotopy analysis method," Communications in Nonlinear Science and Numerical Simulation, vol. 14, no. 2, pp. 473-488, 2009.

[13] V. Aliakbar, A. Alizadeh-Pahlavan, and K. Sadeghy, "The influence of thermal radiation on MHD flow of Maxwellian fluids above stretching sheets," Communications in Nonlinear Science and Numerical Simulation, vol. 14, no. 3, pp. 779-794, 2009.

[14] M. S. Abel, J. V. Tawade, and J. N. Shinde, "The effects of MHD flow and heat transfer for the UCM fluid over a stretching surface in presence of thermal radiation," Advances in Mathematical Physics, vol. 2012, Article ID 702681, 21 pages, 2012.

[15] S. S. Motsa, T. Hayat, and O. M. Aldossary, "MHD flow of upper-convected Maxwell fluid over porous stretching sheet using successive Taylor series linearization method," Applied Mathematics and Mechanics, vol. 33, no. 8, pp. 975-990, 2012.

[16] T. Hayat, Z. Abbas, M. Sajid, and S. Asghar, "The influence of thermal radiation on MHD flow of a second grade fluid," International Journal of Heat and Mass Transfer, vol. 50, no. 5-6, pp. 931-941, 2007.

[17] K. Sadeghy, H. Hajibeygi, and S. Taghavi, "Stagnation-point flow of upper-convected Maxwell fluids," International Journal of Non-Linear Mechanics, vol. 41, no. 10, pp. 1242-1247, 2006.
[18] B. Raftari and A. Yildirim, "The application of homotopy perturbation method for MHD flows of UCM fluids above porous stretching sheets," Computers and Mathematics with Applications, vol. 59, no. 10, pp. 3328-3337, 2010.

[19] J. Alinejad and S. Samarbakhsh, "Viscous flow over nonlinearly stretching sheet with effects of viscous dissipation," Journal of Applied Mathematics, vol. 2012, Article ID 587834, 10 pages, 2012.

[20] T. Hayat, Q. Hussain, and T. Javed, “The modified decomposition method and Padé approximants for the MHD flow over a non-linear stretching sheet," Nonlinear Analysis: Real World Applications, vol. 10, no. 2, pp. 966-973, 2009.

[21] S. J. Liao, Beyond Perturbation: Introduction to Homotopy Analysis Method, CRC Series: Modern Mechanics and Mathematics, Chapman \& Hall/CRC Press, Boca Raton, Fla, USA, 2003.

[22] J. H. He, "Homotopy perturbation technique," Computer Methods in Applied Mechanics and Engineering, vol. 178, no. 3-4, pp. 257-262, 1999.

[23] G. Domairry and N. Nadim, "Assessment of homotopy analysis method and homotopy perturbation method in non-linear heat transfer equation," International Communications in Heat and Mass Transfer, vol. 35, no. 1, pp. 93-102, 2008.

[24] S. Shateyi and S. S. Motsa, "A new approach for the solution of three-dimensional magnetohydrodynamic rotating flow over a shrinking sheet," Mathematical Problems in Engineering, vol. 2010, Article ID 586340, 15 pages, 2010.

[25] M. Sheikholeslami, H. R. Ashorynejada, D. D. Ganji, and A. Yildirim, "Homotopy perturbation method for threedimensional problem of condensation film on inclined rotating disk," Scientia Iranica, vol. 19, no. 3, pp. 437-442, 2012.

[26] D. D. Ganji, "A semi-analytical technique for non-linear settling particle equation of motion," Journal of Hydro-Environment Research, vol. 6, no. 4, pp. 323-327, 2012.

[27] S. S. Motsa and S. Shateyi, "Successive linearisation solution of free convection non-darcy flow with heat and mass transfer," in Advanced Topics in Mass Transfer, M. El-Amin, Ed., pp. 425438, InTech, Rijeka, Croatia, 2011.

[28] S. S. Motsa, Y. Khan, and S. Shateyi, "Application of piecewise successive linearization method for the solutions of the Chen chaotic system," Journal of Applied Mathematics, vol. 2012, Article ID 258948, 12 pages, 2012.

[29] S. Shateyi and S. S. Motsa, "Variable viscosity on magnetohydrodynamic fluid flow and heat transfer over an unsteady stretching surface with Hall effect," Boundary Value Problems, vol. 2010, Article ID 257568, 20 pages, 2010.

[30] S. S. Motsa, P. G. Dlamini, and M. Khumalo, "Solving hyperchaotic systems using the spectral relaxation method," Abstract and Applied Analysis, vol. 2012, Article ID 203461, 18 pages, 2012.

[31] S. S. Motsa, P. Dlamini, and M. Khumalo, "A new multistage spectral relaxation method for solving chaotic initial value systems," Nonlinear Dynamics, vol. 72, no. 1-2, pp. 265-283, 2013.

[32] S. S. Motsa and Z. G. Makukula, "On spectral relaxation method approach for steady von Kármán flow of a Reiner-Rivlin fluid with Joule heating, viscous dissipation and suction/injection," Central European Journal of Physics, vol. 11, no. 3, pp. 363-374, 2013.

[33] M. Renardy, "High Weissenberg number boundary layers for the upper converted Maxwell fluid," Journal of Non-Newtonian Fluid Mechanics, vol. 68, no. 1, pp. 125-132, 1997. 


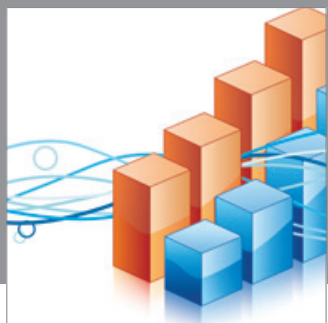

Advances in

Operations Research

mansans

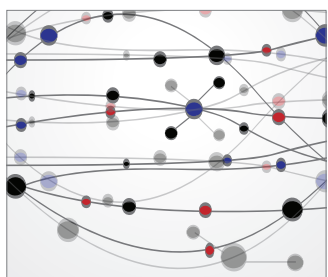

The Scientific World Journal
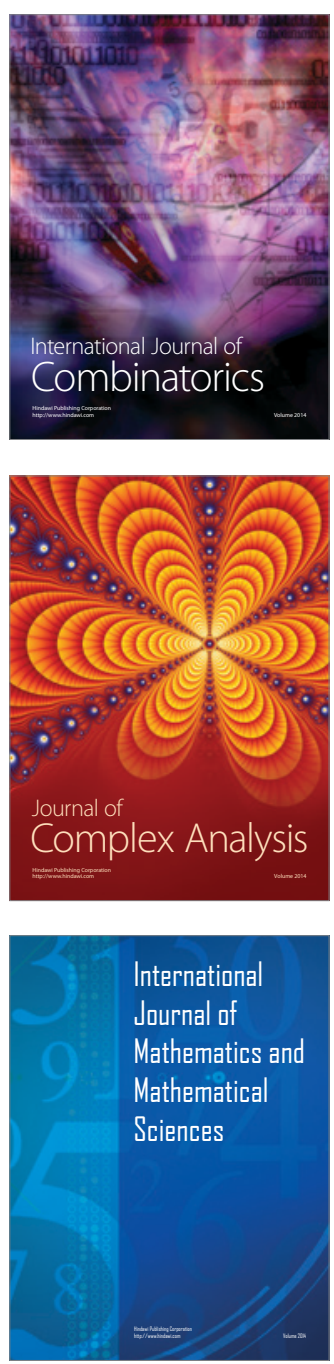
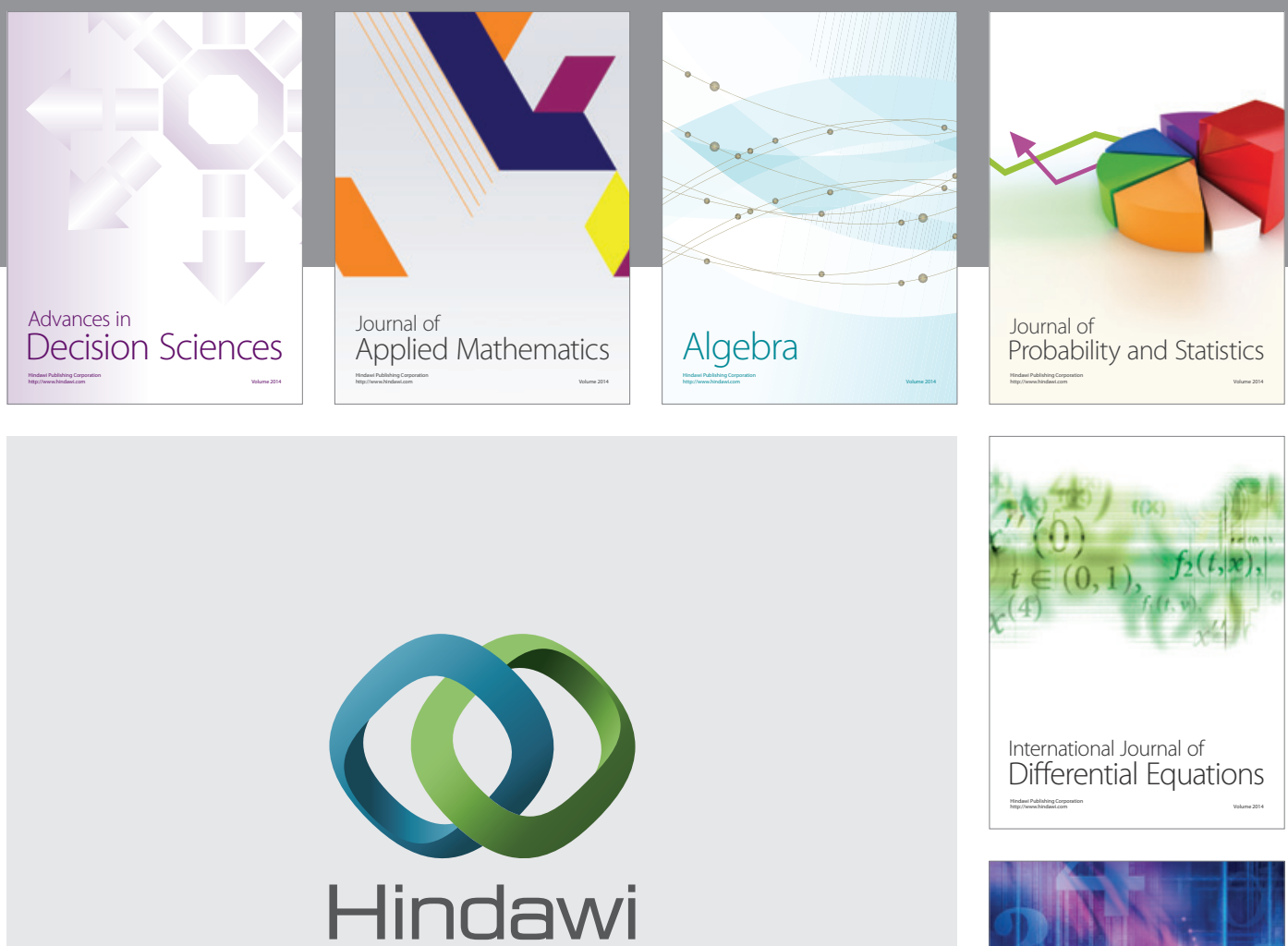

Submit your manuscripts at http://www.hindawi.com
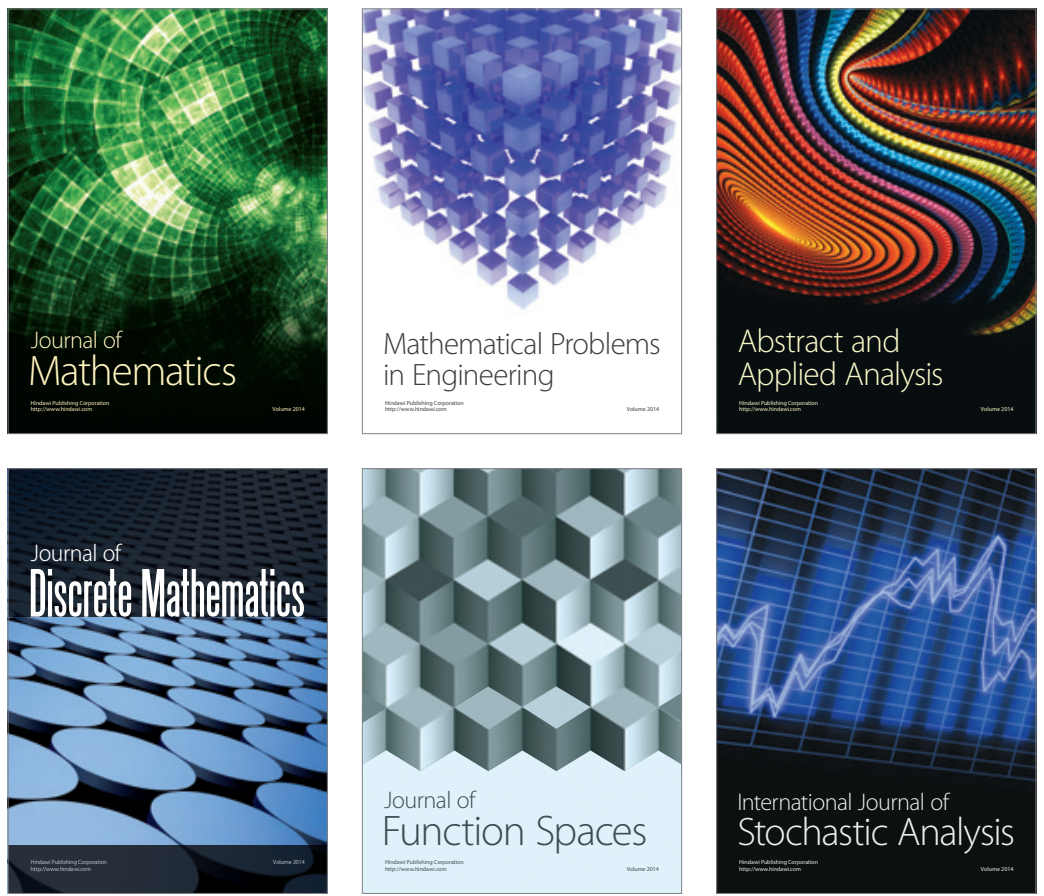

Journal of

Function Spaces

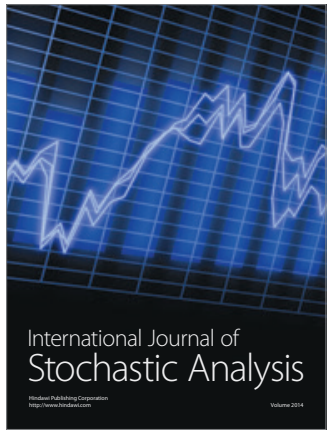

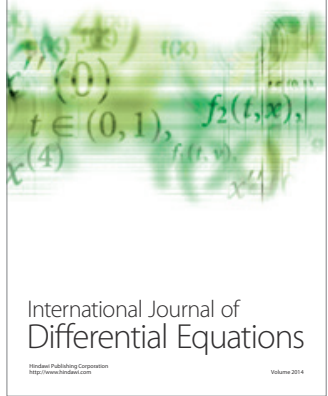
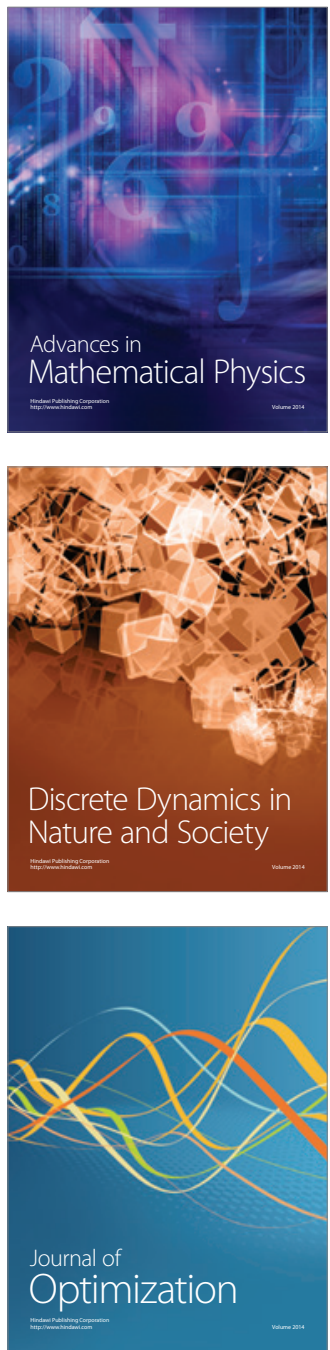